\title{
Time-Neutrality of Natural Laws Challenged: Time Is Not an Illusion but Ongoing Energy-Driven Information Loss
}

\author{
Helmut Tributsch ${ }^{1,2^{*}}$ \\ ${ }^{1}$ Institute for physical and theoretical Chemistry, Free University, Berlin, Germany \\ ${ }^{2}$ Helmholtz Centre Berlin for Materials and Energy, Berlin, Germany \\ Email: helmut.tributsch@alice.it
}

How to cite this paper: Tributsch, $\mathrm{H}$. (2021) Time-Neutrality of Natural Laws Challenged: Time Is Not an Illusion but Ongoing Energy-Driven Information Loss. Journal of Modern Physics, 12, 300-327. https://doi.org/10.4236/jmp.2021.123023

Received: January 21,2021

Accepted: February 23, 2021

Published: February 26, 2021

Copyright $\odot 2021$ by author(s) and Scientific Research Publishing Inc. This work is licensed under the Creative Commons Attribution International License (CC BY 4.0).

http://creativecommons.org/licenses/by/4.0/

\begin{abstract}
It is shown that the time of entropy increase, here called action time, is caused by a dynamically understood energy. It drives time by decreasing its presence per state, that is by abandoning order, information, and creating entropy. This mechanism can be derived from basic principles via the Lagrange-Euler formalism, just considering the properties of really experienced, oriented time and thus abandoning the paradigm of time neutrality. It describes nature driven by a dynamically understood principle of least action, which is identified as manifestation of fundamental irreversibility in nature. This readily explains the second law of thermodynamics and also yields the entropy law for non-linear irreversible thermodynamics: maximum entropy production within the restraints of the system. Dynamic energy-driven time, action time, and time asymmetry is generated via the process of erasing information and liberating its energy irreversibly as heat. It is not an illusion but information-based reality. It is the loss of information to the past and different from clock-time, which is just an artificial scale, using information for tracking real time, action time. Energy-driven fundamental irreversibility of nature can better describe experienced reality and opens the way to understand and finally imitate the self-organizing creativity in nature. It also draws far reaching consequences for understanding quantum physics, gravitation and cosmology as well as biology. From the point of view of irreversibility, nature turns out to be more elegant, simpler and rationally understandable. For the first time, it can be explained in a few words what energy and nature basically represent and why it must have been information, which has started the universe.
\end{abstract}




\section{Keywords}

Time-Invariance, Energy, Time, Entropy, Information, Irreversibility, Self-Organization, Nature

\section{Introduction}

Time-neutrality, that is time translation symmetry and time reversal symmetry, of natural laws with entropy increase towards higher probability as the only practically conceivable time ordinate has shaped the world view of physics. A recent $\mathrm{BBC}$ production with the title: "Are you experiencing time wrong?", dealing with the question, why we are only experiencing time moving forward, while physics says that time can move in either direction [1], highlights the conflict, which the public still feels when confronted with this time concept of physics. It is additionally underlined by a famous historic statement: Einstein, shortly before his death, wrote in 1955 in a letter to the son and sister of his deceased friend Michele Besso: “...the distinction between past, present and future only has the meaning of an illusion, though a persistent one".

The explanation, given by present physics, that natural phenomena develop in direction of increasing entropy and maximum probability can be accepted, because they are evident in nature. But what do they mean in reality and what exactly is their relation to the claimed time neutral fundamental physical laws? The Lagrange function, which is expected to fully describe a developing system and also energy itself are traditionally treated as scalar, non-oriented quantities. They are expected to allow dynamic development in either direction, forward and backward in time. Accepting, that entropy increase in one direction only prevails, anticipates that a phenomenon such as a directional time already exists, because one can observe the increase of entropy in dependence on the time we experience. This phenomenon of entropy increase can, however, not be derived from time-neutral fundamental laws without additional assumptions. Both, the Boltzmann approach (H-theorem) [2] to derive entropy increase, as well as modern Markovian (memory loss) treatments [3] of time-neutral systems abandon information on the past to show that time-neutral natural phenomena finally only continue to proceed in one direction (which only is a mathematical consequence of omitting information, which in a physical system has an energy content). Other attempts to demonstrate time symmetry breaking tacitly assume, besides of coarse-graining (information loss), feedback processes to show development of chaotic, directional properties [4]. They require a distinction between "before" and "after", which in a time-neutral world is not possible (see also later). Statistical reasoning within time-reversible deterministic physics alone cannot explain a time arrow [5]. A humiliating consequence of this situation is that present science, although very advanced in many respects, cannot derive the important second law of thermodynamics, entropy increase in a closed space, 
from time-neutral initial conditions without additional assumptions (not talking about the impossibility to derive an entropy law for non-linear irreversible thermodynamics). So, what is time in reality and why, in practice, it appears to be oriented?

Our clocks need energy to function, but the time they show, the length of their oscillation periods, which are allowed to proceed in form of sequences of oscillations, only depends on natural and material constants. This is also true for the Planck time, the shortest recognized time interval, which only depends on gravitation constant, light velocity and the quantum constant of action. These constants do not produce changes themselves, but their involvement in determining oscillation lengths can be used to generate and measure time periods, which is of course an important technical tool as a scale for a visualization of time in form of progressing and periodically repeating numbers, in form of information. The link to practical life is, as well known, provided by measuring astronomical periods, days, years with clock-time.

This publication attempts to answer the questions, what the time of entropy increase actually is and what relation it has to time neutrality and energy turnover. It further investigates what causes the second law of thermodynamics, what is the time for self-organization in space and in biology and what relation time has to energy and entropy, to which it has been linked in quantum theory via the uncertainty relation [6]. Basing on the identified energy-driven time arrow consequences for understanding nature are discussed.

\section{Results}

\subsection{What Is the Time of Entropy Increase?}

Let us consider a real system which is proceeding asymmetrically, increasing its entropy $S$ from $S_{1}$ to $S_{2}$, for example an expanding gas. Tacitly, present science is assuming that something like a path for time is existing, along which systems can move forward or backward, while respecting intricate behavior within space-time properties. A directional orientation of such processes arises because they are expected to develop in direction of higher probability (lower information content) and are thus imprinting the experienced orientation of time. What is the origin of such a drive? Information is linked to thermodynamic entropy (see later). The difference in entropy generated, the entropy gain, can be multiplied with the absolute temperature $T$ :

$$
T S_{2}-T S_{1}=T \Delta S
$$

and yields the not any more available (entropic) energy $T \Delta S$ (e.g. low temperature environmental heat). We now can ask the question, where this entropic energy came from. Considering the first law of thermodynamics, energy conservation, it cannot arise from nothing, and it could only have been generated from somehow available energy. How did entropy increase with time consequently arise? It was generated from originally available, free energy. Time dependent entropy increase is thus ultimately driven by energy turnover. Since no other 
physical driving element is evident, which may aim at generating entropy, this available free energy must consequently have the property to drive the process, for which time is required. Free energy must decrease its internal order and information content to generate energy in its not any more available, entropic form in a condition of highest probability.

Two different approaches, the presently accepted and the here proposed one, for the same event of entropy production are here confronted. The traditional one (used in present physics) applies a mathematical statistical argument, which calculates, how likely it is that a proposition becomes true. The higher the probability of an event, the more likely it will be that the event will occur. This drives the process along the time path we experience. The second approach, presented here, argues that one is dealing with a physical system, which is subject to physical law, in this case controlled by energy with its properties and its law of conservation. The system must follow natural laws. The author's standpoint: the system concerned is a process determined by laws of physics, the function of which can be described by mathematical formalisms. It is itself not an abstract mathematical process. Therefore, it follows physical regularity towards maximum probability, which can be statistically-mathematically described. Here, physical law has priority over mathematical reasoning. A physical system cannot follow mathematical reasoning without a supporting and direction and rate controlling physical law. The conclusion derived from (1), that a dynamic energy is driving entropy increase, is valid. There must be a physical process aiming simultaneously at maximum entropy (disorder) and energy conservation. It can only be action producing free energy. In contrast to present understanding (energy is treated as a scalar quantity, not interested in performing work) energy should therefore not only have the ability to do work, but also the interest to do it. It should have directional properties, interest in entropy production, in decreasing its information content. Can an appropriate mathematical formalism for such a here expected behavior of energy, which obviously contradicts time-neutrality, be derived from basic principles in support of such a conclusion?

\subsection{Reconsidering Noether's Symmetry Approach}

In order to question time-neutrality in basic laws, it is necessary to deal with the Lagrange-Euler formalism, which is presently used to calculate dynamic and energetic processes in all relevant field of physics, ranging from particle physics to dynamics and Relativity Theory. It is also unavoidable to reinvestigate time invariance considerations which were based on time neutrality and symmetry. Emmy Noether, in 1912, demonstrated that conservation laws can be deduced from symmetry and invariance laws [7]. This way energy conservation was also deduced from time neutrality and symmetry. The mathematical derivation deviates in this case somewhat from standard Noether's theorem since time is not considered to be a generalized coordinate. By totally differentiating the Lagrange function $L$, which should entirely describe a system, reorganizing the relation 
and considering the validity of the Euler-Lagrange-equation, one yields the well-known equation ( $q=$ degree of freedom, generalized coordinate; the point on it indicates time derivative) (e.g. [8]):

$$
\frac{\mathrm{d}}{\mathrm{d} t}\left[\frac{\partial L}{\partial \dot{q}} \dot{q}-L\right]=-\frac{\partial L}{\partial t}
$$

It equates the total time derivation of energy $E$

$$
E=\frac{\partial L}{\partial \dot{q}} \dot{q}-L
$$

with the negative partial time derivation of the Lagrange function (which describes the system via the principle of least action and the Euler-Lagrange equations), or, rearranged, yields:

$$
\begin{gathered}
-\frac{\mathrm{d} E}{\mathrm{~d} t}=\frac{\partial L}{\partial t} \\
\frac{\partial L}{\partial t}=\text { time asymmetry }
\end{gathered}
$$

The partial derivative of the Lagrange function (4a) is considered to express time asymmetry against displacement or translation in time. Traditionally, time invariance against displacement (time neutrality and symmetry with respect to orientation) for the Lagrange function is assumed and time asymmetry (4a) consequently set zero [8]. Basing on time-neutral fundamental laws, $E$ and $L$ are presently considered to be scalar quantities, just numbers. Formulas derived from them can consequently describe dynamic motion both in positive and negative time direction. The time axis is just a preexisting path, which can be used in both directions. Based on such preconditions an assumption of zero time asymmetry ((4a) set zero) was seen justified. The Lagrange function as well as energy, understood as scalar quantities, can accompany a natural system both into the future and into the past. This led to a formal derivation of the law of energy conservation: the right side of Equation (4), time asymmetry (4a), was set zero (compare [8]), since invariance with respect to displacement of the Lagrange function in time was assumed. Consequently, the total derivative of energy is zero. Energy E correspondingly results to be a constant, is conserved in time. This is the well-known conservation law for energy.

Since energy conservation has also been again and again confirmed experimentally, its validity is, of course, undebated (it is later also shown to automatically result from the here derived mechanism). But is this additional confirmation of energy conservation via conventionally "assumed" time neutrality and time symmetry and invariance acceptable and correct? Energy conservation itself does not exclude that free, available energy has other degrees of freedom to show dynamic function on the way to entropy formation and time asymmetry (the total derivative of energy in Equation (4) implies also additional implicit time dependencies, which have to be considered). What is this additional physical property of energy which implements and expresses this time dependence and 
thus the actually experienced directional time flow and time asymmetry?

\subsection{The Correspondence between Dynamic Energy and Time Asymmetry}

Lived time in presence and in the past behaves differently compared with time in the future. We cannot move back into the past and changes performed in the past have an effect on the future. This, however, does not happen in the opposite direction. An energy system, described by the Lagrange function, which started to convert energy in the past, while respecting energy conservation, will have generated different amounts of entropic energy depending on its subsequent position on the time axis. If one accepts these facts about time, which we are experiencing, there is no invariance and symmetry with respect to displacement in time for the Lagrange function. The ratio of free and entropic energy of a system, which a Lagrange function is describing, varies with time and its information (negentropy) content will change, decrease, accordingly along the time axis. The laws or equations that describe a system at time $t$ and $t+/-d t$ are not identical. The behavior is also different in forward and backward direction. Only a behavior in forward direction is actually observed.

Consequently, in Equation (4), the time asymmetry (4a), the partial derivation of the Lagrange function with respect to time, can definitively not be set zero (which would mean zero asymmetry and invariance against displacement in time). During history of physics the convention of time neutrality, the ability of a system to develop in either time direction, forward and backward, has won over experimental facts, deducible from experience with lived time, as just discussed. This practical experience with lived time should have excluded, that time asymmetry as expressed by (4a) in (4) can be set zero. As a consequence, equation (4) gets an entirely different significance. It describes a situation, in which a dynamic energy property (total derivation of energy with respect to time) is related to an asymmetry in time behavior (4a).

What does this here now differently to be interpreted Equation (4) exactly mean for the understanding of energy properties in relation to time asymmetry? Mathematically, Equation (4) equates a partial time derivative of the Lagrange function (time asymmetry) with the negative (decreasing) value of the total time derivative of energy. This means that something decreasing within energy and described by its total derivative is responsible for and driving time asymmetry. A partial time derivative only considers changes with respect to time, omitting their effect on other variables. The total derivative also considers all additional implicit time dependencies (such as on turnover of information, order-see later).

A remarkable consequence of abandoning the experimentally unjustified claim of time neutrality and time symmetry is, that the well-known, established Equation (4), derivable from basic principles, then precisely explains what causes time asymmetry: The necessary consequence derivable from Equation (4) and its correct, experimentally backed interpretation is, that nature is acting in such a 
way, that the decreasing total derivative of energy is related to and defines the degree of time asymmetry as expressed by the partial time derivation of the Lagrange function in Equation (4). The right interpretation of this Equation (4) can only be that the quality of free, available energy changes during energy turnover and thus generates time asymmetry by causing and driving an oriented time. A change in free energy, while energy is conserved, is actually observed during energy turnover. Free energy with a high information content, order (e.g. a chemical fuel), changes into energy with a lower information content (entropic energy (1), e.g. environmental heat), while energy in its totality is conserved.

Since the Lagrange function $\mathrm{L}$ is expected to fully describe the system, Equation (4) expresses that energy (a function of L (3)) with its intrinsic dependencies totally controls the time behavior of the system via its properties. This draws a drastic consequence: nature is not exposed to time neutrality but driven by energy. No new assumption was required, but an experimentally unproven convention (time neutrality and invariance against displacement) dropped in favor of considering experimental facts on lived time, as we experience it. This excludes, as also discussed above (in subsection 2.1.), with different arguments, that a purely mathematical-statistical criterion for attainment of highest probability (and time orientation) is applicable separate from a physical law, which prescribes that. According to (4) it is free energy, with its dynamic property (contained in its total derivative), which is determining the physical law which generates irreversible changes and time asymmetry by approaching a situation of maximum probability. Equation (4) additionally expresses that this dynamic energy property is inseparably related to the degree of time asymmetry (4a). A dynamically behaving energy is responsible for time asymmetry! Via Equation (4) it is related to and causes time asymmetry. Both, energy and time, react as dynamic quantities. Since time asymmetry was deduced from a critical evaluation of time, which we observe and experience, and since the Lagrange formalism is accepted to describe essential aspects of nature, this is a refutation and disproval of the convention of time neutrality and time symmetry within (another disproval of time neutrality follows later).

Here, it is evident, where an obvious, drastic change in understanding nature is needed. Free energy is not a scalar, a simple number, as expected for time symmetry (with (4a) set zero) and able to react in positive and negative time direction. Energy has, in contrast, dynamic, vectorial properties and its behavior is inseparably linked with time asymmetry (according to Equation (4) and meaning (4a)). Relation (4) was strictly derived from established theoretical understanding, but the conventional assumption of time neutrality ((4a) set zero) excluded due to the above discussed experimental facts on lived time. As a consequence, energy must be related to an oriented time, must produce changes over time and must also generate the time asymmetry itself as described by Equation (4). A dynamic energy and an active, directional time are, inevitably, interrelated and linked. Time neutrality or time symmetry (defined as (4a) set zero), which 
has never been supported by experimental evidence, is therefore experimentally disproved and identified as persistent convention, which should be overcome.

The negative sign in Equation (4) indicates that some implicit component of available energy must be able to decrease along with development of time subject to time asymmetry (while respecting energy conservation). The total derivative of energy mathematically considers this implicit dependence on a time dependent variable (below shown to be information). What is ultimately enforcing such a decrease in a property of energy along the time axis? What law the time dependent energy follows is found by rearranging Equation (4) and integrating it to:

$$
-\int \mathrm{d} E \partial t=\int \partial L \mathrm{~d} t
$$

The right side now expresses the time evolution of changes in the Lagrange function, which arise as a consequence of time asymmetry. These are the changes experienced as real time flow, here to be called "action time". What does Equation (5) say in terms of energy? The left side is showing the negative (decreasing) time integral over a total differential energy quantity. It is the decreasing differential "action" (energy times time). This is exactly what the principle of least action is expressing for an infinitesimal section of energy. Equation (5) tells us consequently, that the asymmetric development of the Lagrange function in time (right side), and thus of the physical system, is following and the consequence of the principle of least action (left side). Time flow and changes caused by time are the consequence of fulfilling the principle of least action.

That the principle of least action is applied and works in nature as a fundamental principle is a three centuries long experience in physics. According to Equation (5) it definitely describes a dynamic law, expressing, that, via the principle of least action, nature is fundamentally dynamic and time-asymmetric. A time-orienting change in the Lagrange function follows from a "dynamic" principle of least action. Such a fundamentally dynamic property of this principle is in present physics not considered as such, because energy and time are just treated as numbers, scalar quantities. For this reason, the meaning of the principle of least action has remained enigmatic. In contrast, Equation (5) here implies, that energy has the ability to approach a minimum in some of its qualities (included in the total derivative), following the principle of least action. Energy, subject to the dynamic principle of least action, has to have dynamic properties and, according to Equations (4) and (5), is inseparably linked to and drives time asymmetry. This way, the principle of least action gets a significance, which has never been recognized in the past: the principle of least action expresses that nature is fundamentally irreversible with a dynamic energy generating a directional time, the time we are experiencing. It is a flow of action (energy times time), as expressed in (5). It is not clock-time, which is used to follow this flow of action in an averaged way via oscillating mechanisms, cleared from the drive of energy (see mathematical distinction later).

What is new with an energy, which has dynamic properties? It is in clear contradiction to present handling energy as a scalar quantity with the potential to 
perform work, but no interest in doing it. It is also in clear contradiction with the concept of time neutrality and time invariance against displacement and inversion, which allows to describe energy systems to act both in positive and negative time direction. However, an engineer will understand that energy is needed to produce movement in experienced time direction and the public understanding of "having energy" equally implies dynamic action. Aristotle, who lived in the 4th century BC, already argued along this line: "Time is the measure of a movement that takes place from a before to an after". His idea of time did not describe any illusion, but he recognized experienced movement, energy-driven reality, as an expression of time. He described action as generated by a stone rolling down a hill (following the principle of least action) and stated that passing time is linked to such movements (as expressed in (5)). Since movements are linked to energy turnover, time is linked to a "dynamic" energy. All together makes sense because it reflects reality.

Let us recall, what could be learned up to now in order to find out what decreasing energy property is accompanying the drive, responsible for the time-orientation in free energy. When energy is responsible for the dynamics of a system, described by the Lagrange function L, then Equation (4) also expresses that there is no time invariance against displacement in time ((4a) cannot be set zero). An energy system therefore develops in direction of increasing entropy and generates time asymmetry. Equation (5) in addition tells that this occurs via the principle of least action. This means that energy acts as a dynamic variable and that the time generated is directional (which is also experimentally observed). It also describes that the time-related properties of a system (described by $\mathrm{L}$ ) are exclusively determined by dynamic time dependent properties of energy (which presently is excluded by the concept of a scalar energy quantity). This supports the arguments presented above in subchapter 2.1. Energy consequently not only has the property of being conserved (as experimentally proven, and later confirmed for the presented formalism), it simultaneously has an additional dynamic time dependent property itself (considered in the total derivative of energy in Equation (4)). The same is then, of course also true for the Lagrange function. Both have to be considered to act as dynamic variables, entirely in contrast to their present role as scalar quantities. By becoming time oriented they abandon the world of time-neutrality. Energy is not consumed, not decreasing in quantity. What time dependent property contained in energy and considered by its total derivative in Equation (4) is then actually, between free energy and entropic energy, changing and driving time? During entropy production information is abandoned and energy is involved. What is the role of information in such an energy conversion process?

\subsection{Entropy Formation and Energy Driven Information Loss}

It is well known that thermodynamic entropy $S$ and information entropy I are related via ( $\mathrm{k}$ is the Boltzmann constant) (e.g. [9]). 


$$
S=k \ln 2 I
$$

An increase of entropic energy $T \Delta S$ is, since energy is conserved, linked to a corresponding turnover and decrease of free energy $\Delta E$. An increase of information entropy I is linked to a decrease (negative sign) of actual information $I_{\text {act }}$. Replacing thermodynamic entropy $\mathrm{S}$ for information entropy $I$ (6) and considering that an increase of information entropy I is linked to a decrease (negative sign) of actual information $I_{\text {act}}$, the working ability of available energy can be calculated. It is subject to a decrease in contained actual information $I_{\text {act }}$ (within free energy) according to the following relation:

$$
\Delta E \rightarrow T \Delta S=-k T \ln 2 \Delta I_{\text {act }}
$$

In this relation $k T \ln 2$ is the energy needed to activate 1 bit. It is again liberated as thermodynamic heat (entropic energy), when erased. With the above considerations (subchapters 2.1. and 2.2.) identifying free energy as the origin and driving source of entropy increase and of oriented time, it means that the turnover of free energy is linked to a decrease of information. During an energy conversion process, energy itself is conserved, but information (order) within the original free energy is decreasing. This decrease in the information content is considered in the total time derivative of energy (Equation (4)) and is therefore directly linked to the generation of time asymmetry. The product is chaotic, entropic energy with a negligible level of order or information left. This abandonment of information from free, available energy, during production of entropic, chaotic energy, is responsible for the generation of time intervals which are adding to time asymmetry. The process of entropy increase in combination with a progressing time can therefore be attributed to a free energy with the property of aiming at a reduction of presence per state, which is equivalent to a diminution of order, information, as described by relation (7). Practical examples are heat dissipated into the environment or an excited molecule releasing energy in smaller quantities via various pathways.

Such a decrease in information, which free, available energy, $E(t)=E\left(I_{\text {act }}(t)\right)$, experiences during energy turnover, on its way to entropic energy, is obviously considered in the overall energy balance and in energy conservation. Since the work of Szilard [10] it is known that the minimum energy required to store 1 bit of information is $k 7 \ln 2$. Landauer [11] has shown that erasure of 1 bit of information generates heat and increases thermodynamic entropic energy by $k T \ln 2$. Free energy already contains the information (e.g. stored in chemical bonds of fuels, which create the ability to do work) and when erased its energy is still there as entropic energy in the form of low temperature heat. Energy conservation is therefore also fulfilled for the total time derivative of $E(t)$ in Equation (4), which expresses, how dynamic energy drives time asymmetry via decreasing contained information and increasing entropy. The information lost during entropy formation (7) was originally still available for free energy and its turnover is the origin and thrust of energy-driven changes. 


\subsection{What Exactly Is Experienced Time?}

Since science has based natural laws on time neutrality and considers time to be an illusion, numerous attempts have been undertaken to make real, experienced time more understandable (e.g. [12] [13] [14] [15] [16]). They yielded deeply rooted considerations, studied wise comments on time since antiquity and discussed intelligent statements from experience, but essentially remained futile, because explaining lived time as a consequence of time neutrality and from the viewpoint of relativistic time paradoxes turned out to be practically impossible. For most observers, it remained therefore an open question what experienced time actually is.

According to the considerations presented here, science itself causes the problem. Science has simply to drop the persistent convention of time neutrality and time neutrality and symmetry in fundamental laws. It is a heritage from historic times, when formulation and understanding of dynamic laws were still too complicated. The time of entropy increase and the entropy increase itself are not compatible with time neutrality and the concept of time as an illusion. Time is caused by free energy, which has to be considered dynamic and time-oriented. Subject to this new interpretation time is the

"energy-driven flow of action observed in nature", as expressed by relations (5), with $\mathrm{L}$ and $\mathrm{E}$ here being dynamic variables. Action time (the flow of action subject to the principle of least action) as described by (5), is progressing in an inhomogeneous way, within large and small processes, which may proceed simultaneously or in consequence. Since energy, via the processes activated in generating action time, thereby loses information (7), time is also the

"ongoing loss of information about the past".

Progressing time is, in fact, a process of information loss due to energy turnover.

Since action-time, with its turnover of action and information respectively, is, in nature, occurring locally and temporarily inhomogeneous, clock-time is to be used and calibrated as a scale for defining and measuring an averaged progress of action-time. Also, our brain may be doing this with changes going on in the environment.

The energy-driven time, "action time", is directional and makes natural processes fundamentally irreversible. Energy, consequently, by generating action, via the principle of least action (relation (5)), drives a time, which is oriented and provides a flow of information turnover. This can be mathematically derived by inserting the expression (7) describing the information to be lost from active, free energy on its way to entropic energy into Equation (4) and considering that free energy still disposes of this information (changed sign). This relates the information-based quality of energy and its turnover to asymmetric time evolution. One yields: 


$$
-\frac{\mathrm{d} E}{\mathrm{~d} t}=-\frac{\mathrm{d} E}{\mathrm{~d} I_{\text {act }}} \frac{\mathrm{d} I_{\text {act }}}{\mathrm{d} t}=-k T \ln 2 \frac{\mathrm{d} I_{\text {act }}}{\mathrm{d} t}=\frac{\partial L}{\partial t}
$$

This formula (8) shows that asymmetric time evolution, the partial time derivative of the (dynamic) Lagrange function $L$, is directly related to the decrease of a property of energy $E$, which turns out to be the loss of information $I_{\text {act }}$ (including its energetic value $k 7 \ln 2$ ) according to Equation (7). Again, $k 7 \ln 2$ is the energy for creating one bit of information, which is then released as entropic energy (e.g. environmental heat) when the information is again erased.

When free energy is turned over on its way to entropic energy, energy itself is conserved, but information is lost. Since information stored in energy itself has an energy content which is then reappearing as entropic energy, turnover of information works in accordance with overall energy conservation. Energy is not lost in the process and the reason for energy conservation, which was previously veiled, becomes obvious.

How are we experiencing time flow around us? Energy turnover is linked with information turnover (8). When a new energy source is appearing in parallel, more information will initially be there, which equally will be gradually lost during energy turnover, and this will go on with more and different energy sources in the environment or the living body. An observer experiences, via the energy-phenomena observed, time-displaced sequences of images of varying information content (Equation (8)). It is like seeing successive complex images adding to a movie. Experiencing time is actually like seeing a movie. Energy driven, changing information turnover is a key to understand passing time as a reality, an information-based energy-driven reality. Since energy turnover proceeds under permanently varying conditions, such information will typically be different and characteristic for past, presence and future. A distinction between them, as in daily practice, is clearly possible and can be documented via information. In addition, turnover of action and information (including erasing it) respectively (Equations (5) and (8)) are invariant during relativistic transformation. We are not dealing with an illusion and relativity paradoxes, as found for clock-time, disappear for action time.

This conclusion obviously contradicts the above-mentioned statement by Einstein on time (clock-time) as an illusion. According to arguments presented here experienced time is not an illusion (nor is it clock-time) but is information-based reality! A continuously generated flow and turnover (erasing) of information, which accompanies the parallel flow of action, generates action-time, which is not an illusion.

A block-universe understands time differently: Relativity Theory is based on time-neutral natural laws and Einstein calculated and commented on clock-time, which is not energy driven, not the real time accompanying changes discussed here, but a normalized scale, contradicting equation (4), which defines the origin of time asymmetry. Clock-time is a kind of frozen time, a strange contrast to the time really experienced. It was nevertheless used to design the fourth dimension of space-time and was exposed to relativity considerations (Einstein insisted that 
time is clock-time). According to the formalism developed here clock-time is just a scale and has not the potential to tell us how energy converting systems are actually behaving in their own dynamic way.

During the rise of Relativity-Theory the Nobel prize winning French philosopher Henri Bergson attacked Einstein accusing him of "spreading metaphysics over science by describing time and its formulas as a time not lived by human experience". Einstein replied that "there is no (separate) time for philosophers" [17]. Here it was shown that such time for philosophers (and reflecting people) exists. It is action time, which is not subject to relativistic changes (see later). The considerations developed in this paper give Bergson right. Time (clock-time) as an illusion does not support and explain real life. Action time (5), which is described here as the experienced, energy-driven, information erasing time phenomenon (8), is clearly different from clock-time, which is based on a different foundation but, of course, useful and necessary for registering and calibrating averaged changes. Since its information comes from and involves energy, action time (5) communicates the message of energy phenomena. Clock-time, on the other hand, is not the real time, but a kind of "alias". It has nothing to do with energy turnover and uses combinations of natural constants and material properties to measure, via chains of oscillation periods, an averaged progress of energy-driven real time flow (action time) in the environment, ultimately calibrated for practical use with periodical astronomical phenomena.

\subsection{Real Time (Action Time) Means Erasing Information, Clock-Time Just Using It}

What is the mathematical relation between real time (action time) and clock-time? In order to better understand what action time, defined as time evolution of changes in the Lagrange function, means and what its relation to clock-time is, the rearrangement and integration of formula (4) to yield (5) should now be repeated with formula (8). One obtains:

$$
-\int \mathrm{d} E \partial t=-\int(k T \ln 2) \mathrm{d} I_{\text {act }} \partial t=\int \partial L \mathrm{~d} t=\text { action time }
$$

It describes action time as the loss of information $d I_{\text {act }}$ to the past, but multiplied with the energy $E^{\prime}=k T \ln 2$, which is liberated when erasing one bit and turned over into entropic energy (heat). This means that free energy has to be turned over and thereby information to be erased to generate action time. It has the dimension of action (energy times time, where time has the meaning of clock-time) and is relativistic invariant.

In order to obtain clock-time, action time (with the dimension of a flow of action) has to be divided by the energy turned over per bit, $E^{\prime}=k T \ln 2$. The result is shown in the following equation:

$$
-\int \frac{\mathrm{d} E}{E^{\prime}} \partial t=-\int \mathrm{d} I \partial t=\int \frac{\partial L}{E^{\prime}} \mathrm{d} t=\text { clock-time }
$$

Clock-time is, as deducible from relation (10), providing information, but this information is not turned over, erased. It is simply, since energy $E$ and Lagrange 
function L were neutralized as dynamic variables, used as a scale providing information. It is also used in this simple function for defining the fourth dimension in space-time. No energy turnover and corresponding information loss or erasure is involved in this case. No time asymmetry is involved any more. Clock-time is therefore only useful for registering an averaged, energy-driven progress of action-time (9). It is just a tool for measurement and as a scale subject to relativistic changes. These changes have, however, no significance for energy turnover and time asymmetry, as described by (4) and (5), which follow action time (9). In conclusion, it can be said that action time (9) flows, driven by the principle of least action, while clock-time (10) is frozen, just a scale, invented by humans.

\subsection{How Humans Are Experiencing Time}

Let us now consider how humans experience time and were and still are dealing with it. Many energy conversion processes are all the time occurring in the environment as well as in the human body itself. Geological processes, life activities, weather phenomena and social events are continuously proceeding and consuming (useful) energy. This occurs via a turnover of information (8), which is thereby finally erased and liberated as heat.

Physically, this means that information from the environment or the human body, which was present before, is gone and new information, from ongoing additional energy turnover, is available. Humans and other living organisms have learned to experience the phenomenon of vanishing older and newly made available information from their environment as time flow. Since it turned out to be crucial for their living activities humans attempted to measure and subdivide time and found that periodical natural phenomena were especially useful for this purpose. They followed and studied the movement of the sun and marked the cast shadow. They also studied the movement of the moon. This resulted in useful calendars for daily and annual activities. Since Kepler empirically formulated his laws and later Newton provided the mathematical tool to calculate them it is known that the formula for the time periods of cycling planets and satellites only depend on system constants and natural constants. They do not contain any reference to energy turnover.

Later humans developed artificial clocks, which are also based on periodical phenomena (pendulum movement, quartz oscillation, atomic transition), which require energy for visualization, but the time periods they apply have again nothing to do with energy. Clocks, like planetary cycles, provide information (10) useful for monitoring changes in the environment. The information used in this case is however not erased, but allows to monitor energy driven action time (9) in an averaged, calibrated way via the provided scale.

Different from energy-driven real time (action time), clock-time, as a scale, follows its own laws. Itself it does not reflect the flow of action and of information lost to the past, as seen from (10) in comparison with (9) for action time. 
Clock-time is a kind of standardized, energy-neutral scale (10), applied for monitoring the ongoing energy-driven erasure of information in the environment (9).

It is the author's conviction that mayor problems in physics started to arise, when clock-time, with concepts basing on time neutrality and incomprehension of the dynamic role of energy (4), was identified with action-time, the really lived time: Einstein commented: "time is what the clock shows". It would be more correct to say: "time is what clocks partially imitate".

As a pure scale, not involving erasure of information, clock-time is changeable, subject to relativistic transformation, with conclusions to be drawn, which are presently shaping the concept of our universe. They are known to be to a significant extent paradox and counterintuitive. Examples: Within the presently favored block universe the most distant galaxies, already approaching light velocity, will be experiencing nearly time standstill (and higher life there expecting this also for our own galaxy). In contrast, the here derived energy-driven action time (9) is invariant. Life in these galaxies, subject to the same energy laws, will proceed equally fast there. The mentioned notion of galaxies wandering off with close to light velocity (velocity even doubled (!) when referred to opposite universe position) may also be treated with caution, since time-neutral quantum physics could not properly consider irreversible energy loss (red-shift) for spreading photons via entropy generation (see later). Time travel, with all its paradoxes, is inevitably fiction as well. Energy consuming travelers would have to travel with action time (9). It is relativistic invariant, functioning via erasing of information and not with frozen clock-time (10), within which they would only accompany information into the past or into the future. This information from clock-time, as a scale, can be compressed or stretched, because subject to relativistic transformation, and therefore causes counterintuitive paradoxes, which have nothing to do with reality.

\subsection{Information-Based Proof of Time Asymmetry in Energetic Processes}

After such far-reaching conclusions, which indicate that clock-time is not expressing essential energy-related reality but only acting as a scale, a reevaluation of the responsible basic claim-absence of time neutrality in natural laws-is appropriate (test of validity of Equation (4) as drive for time asymmetry). An additional critical test is now possible for verification whether abandoning time neutrality and time displacement invariance is justified and consistent with present knowledge about energy behavior. For this purpose, one can use the derived Equation (8) to evaluate its deeper meaning. By insisting on (experimentally not supported) time-neutrality and time-symmetry (the partial derivative of the Lagrange function (4a) in (8) set zero) it follows from (8) that $\mathrm{d} I_{\text {act }} / \mathrm{d} t$ then equals zero and as a consequence acting information $I_{\text {act }}$ in free energy would always stay constant. Acting information would thus remain conserved during energy turnover. Such a conclusion, derived from basic Equations (2) to (4) while just still insisting on time neutrality of natural laws, is entirely in conflict 
with our understanding of energy turnover from valuable free energy (high order, high information content) to not any more valuable entropic energy (low order, low information content). It thus disproves the here hypothetically assumed time neutrality on experimentally verified grounds. It is entirely incompatible with our understanding about energy. It is also in conflict with our knowledge about information, which is not a quantity, for which a conservation law is known or even applicable.

In turn, the experimental fact that information is erased, not remaining constant, during energy turnover proves time asymmetry and disproves time symmetry ((4a) set zero) according to Equation (8).

In a time-neutral universe, as it is claimed to exist today, energy turnover, as we know it from long-term experience, would simply not work, since free, available energy must lose information on its way to entropic energy (e.g. low temperature heat). Time neutrality is thus entirely inconsistent with known, relevant properties of energy and information. Formula (8) also indicates that information turnover is essential for energetic processes and is the key towards performing work. The here presented test, together with the above discussed asymmetry of lived time, again underlines the justifiability of challenging fundamental time neutrality as a claim which contradicts reality and contributes to an imaginary model of nature and our universe.

In this context, it is appropriate to ask what it exactly is what makes energy with high information content (free energy) much more valuable and active than the same amount of energy with low information content (entropic energy). It is the intricate structure of chemical bonds, the profound and complicated physical nature of electromagnetic or gravitational fields, the complex electron distribution in excited molecular states, which get mechanisms to work via the elaborate information they contain and make available. Such information is lost on the way to entropic energy, because information is erased and its energy content released in form of not anymore useful entropic energy. It is obvious that it is the quality of information contained in energy which is determining its value as energy source. This information is lost and erased during energy turnover, used to perform work or dissipated as heat, and this process contributes, according to Equation (9), to the progress of real time, action time. Real time, action time, is an energy-driven information phenomenon which, because it strikingly designed the environment, has shaped the existence of living beings. Clock-time (10), as a frozen scale provides information, but does not erase it. It can be used to track, calibrate and measure action time, which may proceed inhomogeneous in time and space (9), from outside the information erasing systems. But itself clock-time cannot give deeper insight into fundamental properties of energetic processes which generate experienced time.

\subsection{Thermodynamic Entropy Laws Follow without Additional Assumptions}

According to relation (8) abandonment of actual information, erasing it, from 
energy systems, which reflects the occurring changes, is thus related to asymmetric time evolution (4a) and entropy increase (7). Progressing time, action time, is linked to an ongoing erasing and deletion of information. It is obvious, that the important second law of thermodynamics, the time dependent increase of entropy in a closed space, automatically follows from such energy-time properties, as expressed by (8) and considering (7). The information content in the system decreases (entropy increases) and generates time asymmetry while energy in its entirety is conserved. It is a dynamic energy and not higher probability, which is ultimately responsible for time orientation. Via relations (8) and (9) this is a straightforward result. It is significant to point out again, that the presently favored time-neutral formalism does not allow derivation of this important empirical second law of thermodynamics without additional assumptions. Here, the difference simply is, that the paradigm of time neutrality ((4a) set zero, which is an experimentally unproven convention) was abandoned considering the known properties of lived time, and taking into account that, while the total energy is being conserved, the information, lost due to entropy generation, must have originated from free, available energy. A derivation of the second law of thermodynamics from basic principles (Equation (4)) succeeded, though with the message, that nature is fundamentally irreversible.

The flow of information, abandoned and erased from an energy converting system, which aims at a decrease of energy's information content (order), is also the key to distinguish between a "before" and an "after" (with different information contents) for facilitating feedback in self-organization processes. Because of the relation between information turnover and time flow (8) it is information which ultimately links past and present (a distinction which is not available in a time-neutral, time-invariant world) during build-up of local order at the expense of entropy production. It is the energy-driven, information handling action time (9) which pushes open the door to the creative world of non-linear irreversible thermodynamics facilitating impressive terrestrial and galactic phenomena as well as biological nature and life. It should, by allowing better understanding, at the longer term, also help to develop bio-analogue energy and material technology, which has been so successful in nature, but is subject to complex self-organization processes, which fail subject to time neutrality. What entropy law do such self-organized non-linear processes follow?

Equation (8) with Equation (7) state that for dynamic systems far from equilibrium a reduction and erasing of information, equivalent to entropy production, becomes rate limiting. This has a significant consequence. Self-organized, feedback-driven systems will accelerate this rate and finally maximize the rate of information loss (entropy gain) within their restraints. It means they follow an entropy law and an aim:

"maximum entropy (energy) turnover within the constraints of the system".

A hurricane will aim at harvesting maximum energy from the sea environment and will grow in power accordingly, but slow down after landfall due to 
changing constraints.

This entropy law for non-linear irreversible processes gives important new insight (see also discussion later), which could up to now not be derived from reversible thermodynamics, due to rate limitation of chemical processes via statistically occurring collisions with (scalar) activation energies to be surpassed (of course still valid as approximation near equilibrium).

Biological systems must follow this aim of maximum entropy production as well (an aim is hitherto strictly denied in biological science), but its implementation is not only balanced by given restraints of the systems, but additionally guided and controlled by the evolved and superposed genetic code. They are teleological systems due to these feedback processes involved, as already recognized in 1943 for cybernetic systems [18] (e.g. regulatory and purposive electronic devices). They are subject to a purely materialistic purpose generated by the systems themselves.

Why was it so easily possible to derive the second law of thermodynamics and the entropy law for non-linear irreversible thermodynamics from dynamic energy-time considerations (Equations (4), (7) and (8))? These laws are dealing with energy systems developing in time. Consequently, a dynamic relation between energy and time was needed to understand and describe them. Equation (8) could provide it, not however a concept of time-neutral natural laws with a scalar energy quantity, which has no interest reacting in time, which as clock-time in addition was treated as frozen.

\section{Summary and Discussion}

\subsection{Time Is the Track of Energy-Driven Information Loss}

Physics today, while insisting on time-neutrality and time-neutral fundamental laws, takes note of the phenomenon of entropy increase over time as a striking natural process and accepts that time practically proceeds via mechanisms, which aim at maximum probability and measures it with clocks. The time response of clocks has nothing to do with energy turnover. The time progress they measure only expresses sequences of time intervals of oscillating systems the oscillation period of which is determined by natural constants and material parameters. This clock-time, embedded and manipulated within General Relativity Theory, became a crucial element in constructing a block-universe, in which time is considered merely to be an illusion, depending on relative movements [19]. Equation (10) explains, why clock-time is indeed an illusion: The information it expresses is just a scale. It does neither reflect changes nor orientation, which energy turnover traces, which requires erasing information, which releases kTln2 in entropic energy for each bit, as relation (9) describes for action time.

As suspected at the beginning of subchapter 2.5., the claimed time neutrality for natural laws and time paradoxes from Relativity Theory were essential stumbling blocks on the way to understanding time. The considerations presented here, which rely on a re-interpretation of the Lagrange-Euler formalism on the 
basis of experimental facts on experienced time, derive, that this experienced time is an expression of a fundamentally irreversible nature. Time is generated and pushed by energy (expressed by Equation (4)), which is causing movements and changes, and is not at all an illusion. Equation (4), when expressed by "dynamic" quantities of Lagrange function and energy (and not by scalar quantities, as characteristic for a time-neutral world), is the origin of an energy-driven time, which is oriented, and thus the basis of time asymmetry. Free energy, by decreasing its order and erasing information, and thereby producing entropic energy, generates this oriented time as a flow of action, action time (Equation (5) and (9)). This energy-driven time flow is caused as a process of erasing information on the past. During generation of action-time via such a mechanism, intervals, measurable as passed clock-time, are apparently inevitable. They simply show up during the process of formation of energetic products of lower information content (entropic energy $T \Delta S$ ). Time (measurable as clock-time) is required for erasing information and converting energy into entropic energy, environmental heat.

On the basis of such results time is not a pre-existing time-neutral axis in positive and negative time direction, as presently assumed. It is an oriented dimension created by continuous information turnover as a consequence of energy turnover, or, more precise, by abandonment and erasure of information contained in available energy. In this respect time is clearly an information phenomenon. Its function is dealing with and providing information. Real time in nature (here called action time (9)) is the ongoing process of information loss to the past and ultimate expression of nature's dynamics and creativity. Such complex information processes are not accessible for clock-time, which is a designed and calibrated information scale (10) useful only for keeping track of action time (9).

Energy-driven, information handling processes generate "action time" (5) (9) and make up the changing world we are seeing around us as a sequence of changing information images (expressed by (8)). Clocks, working via oscillations determined by natural constants and material parameters, are however needed to monitor action time in an averaged and calibrated way. They are constructed not to measure the flux of abandoned information (action time) during "lived" time, but simply provide information on changes in form of a time scale (10). These clocks measure the progress of action-time, but do not any more give access to relevant properties of energy systems. When atomic clocks are sent flying around the globe the observed microsecond shift [20] is real, but here expected to be an effect of calibration and relativity changes due to changed parameters during travel affecting atomic oscillations only. It is not a change in real time, action time, which is an energy phenomenon and of different nature. Technical installations, which work with clocks, have, of course, to consider such deviations, when required.

When the real time in nature, action time (expressed in (5) (9)), is to be transformed to a moving system, the energy-driven flow of action (or turnover 
of abandoned, erased information) has to be transformed, and action as well as turnover of abandoned information remain invariant. Relativity paradoxes disappear for energy converting systems. The clock-time of the block-universe [21] is not the real time-it is indeed an illusion (as claimed by its proponents themselves), an artificial time scale shaped by natural and system constants defining oscillation periods and subject to characteristic relativistic transformation. It has, as its mathematical description (10) clearly shows, neither to do with time asymmetry (defined as the partial time derivation of the Lagrange function (4a)), nor with energy-driven systems (the total time derivative of energy). In order to describe relevant natural systems, the time must get the property of thereby simultaneously abandoning information as action-time does (9) and energy converting systems do.

The scientific strategy applied here to question time neutrality was based on a simple, transparent procedure: starting from the established Lagrange and Euler-Lagrange formalism (Equations (2)-(4)) and energy-entropy notions (1), the time-symmetry claim (4a) for Equation (4) was questioned and disproved basing on experimental facts on lived time. Time asymmetry, the partial derivation of the Lagrange function with respect to time (right side of relation (4)) cannot be set zero because this contradicts our daily experience with time and with energy converting systems reacting in time. As a consequence, Equation (4) cannot be applied to justify a time-neutral nature supporting energy conservation only. It expresses an energy-driven evolving nature, which leaves a time track of abandoned information.

Equation (8) was, in subchapter 2.8., also used to show that time neutrality contradicts established experimental knowledge about the function of energy turnover. Information is not conserved between free and entropic energy, but has to change in order to facilitate energy turnover. In turn, the need for information turnover during energy conversion contradicts time neutrality of natural laws, as seen from (8).

It is an interesting discovery, that the frequently used Lagrange-Euler formalism for the description of dynamic mechanisms included the possibility to derive an energy-driven irreversible nature as done here by insisting on the experimentally observed, actually existing time asymmetry in Equation (4). The only prerequisite would have been to accept the reality, that experienced time is indeed asymmetric ((4a) not zero). Then equation precisely (4) tells, that energy is actually driving irreversibility and changes, what every engineer would have understood to be an experimental fact. This did not happen and shows, how strongly rooted the historically grown convention of time-neutrality and time symmetry in natural laws was and still is. It also led to the (here considered erroneous) conviction that the frozen clock-time (the "alias" for measuring real time flow) itself is determining reality. It cannot, since it does not trace the actual changes occurring during energy turnover, but simply acts as an information providing scale, suitable for measuring changes only. 
Energy (free, available energy) has a time dependent and time driving component (considered in the total derivative of energy in (4)), which can be identified as its content of information (order), which is being gradually erased and correspondingly decreases during entropy formation. This conclusion is supported by the rearranged Equation (5), which then expresses, that the evolution of the Lagrange function is determined by the decreasing differential least action. This fulfillment of the principle of least action, by energy, which is an experimental fact, equally requires that energy has a dynamic property. Free, available energy generates oriented time, here called action-time, as a flow of action or a flow of information loss (as seen from (7) and (8)). As Equation (5) shows, the principle of least action is specifying the dynamic mechanism through which energetic processes and thus erasure of information are implemented, but with Lagrange function $\mathrm{L}$ and Energy $\mathrm{E}$ acting as dynamic variables.

\subsection{What Would Be Different in a Fundamentally Time-Oriented Nature?}

The attempt here, to bring the established paradigm of time neutrality, which could never be supported by direct experimental evidence, into critical discussion, is a mayor challenge, because it is deeply rooted in physics and anchored in much-tested theories. Besides of the arguments and proofs presented here it will be necessary to demonstrate, that a transition from a time-neutral to a fundamentally directed, irreversible nature, brings substantial advantages for general physical understanding in terms of simplicity, logic and meaningfulness.

Such evidence partially already exists as a result of recent investigations, by the author, originally aimed at investigating and questioning the origin and necessity of the rising number of paradoxes and irrational theoretical models in concepts of physics, which were incorporated into "scientific understanding" during the past century (e.g. non-locality, uncertainty, zero-point energy, energy from nothing (Big Bang), expanding empty space, relativistic paradoxes, time as illusion, time-travel, bent empty space, multi-dimensions, multi-universes). The conclusion reached from such an effort was, that the irrational assumptions and paradoxes were ultimately assumed and required in response to the (claimed, here considered incorrect) time-neutral character of fundamental physical laws. If, on the other hand, a dynamic energy is allowed to push time, action time, as underpinned in this publication by deriving the dynamically understood Equations (4), (5) and (8), existing experimental reality supports a quite different irreversible, self-organizing nature and universe [21]-[26]. It is rational, requires less assumptions, is simpler in structure and, due to its entirely logic nature, more convincing. It appears to be, as also the simple derivation of important thermodynamic entropy laws (from (8), subchapter 2.9.) underlines, clearly favored by Ockham's law of parsimony.

The author is convinced that he has proven that time-neutrality of fundamental laws is not compatible with our long-term experience with energy as well 
as information. This has the consequence that important theories basing on time-neutrality (the possibility to displace systems in time homogeneously in positive and negative direction) are either wrong, superfluous, or just applicable as limiting cases. Abandoning time neutrality, on the other hand, means that new theories and concepts describing nature will become possible. In order to rise interest in critically questioning time-neutrality and in looking at consequences, a few examples may show the contours, scientific prospects and consistency of the alternative, of an energy driven, time-determining self-organizing universe, based on the dynamic understanding of energy and time as discussed above:

- Quantum states become dynamic phenomena and have to be understood differently. Particle and wave are not any longer equivalent and simultaneously present in time neutrality. Instead, the spatially extended wave results from the particle, but requires information on matter for the reconversion into the particle and to be in balance with it (analogy to concept of Maxwell demon). This information (on matter) is activated in the quantum state and turned out to be the missing information needed to overcome quantum paradoxes [21].

- Matter is self-organized energy. This immediately explains the known proportionality between energy and matter (relativity considerations are not required). A new and different way of looking at the large and complex family of elementary particles is possible (comparison with self-organized, inactive viruses) [22].

- The "information on matter" attributed to dynamic quantum states and present around them seems to fulfill all attributes of gravitation and provides the link between the quantum world and cosmology [23]. Gravitation is information on matter. Satellites are teleguided by this information (gravitation), when following the principle of least action.

- The ongoing turnover of quantum states between particle and wave, via information they contain, explains the always constant remaining light velocity (light particles, including their velocity, are recreated via information in any relative system). The same information on matter explains gravitation itself. A postulated bent space-time, which enforces exactly these two natural phenomena via the General Relativity Theory [27], is not at all needed and manifestations attributed to it may find different explanations [24]. This realization that the Theory of Relativity is not required is the more relevant, since above considerations (subchapters 2.6. and 2.7.) have shown, that clock-time, used in it, is not the real dynamic action time (9) to which energy systems are exposed, but just a frozen technical time scale (10).

Relativity Theory, based on the assumption of time-neutral natural laws and clock-time, is presently generally accepted because claimed to have passed essential experimental trials. Tests, which differentiate between a space-time universe and an energy-driven universe, subject to action time, are unavoida- 
ble. They could experimentally concentrate on the distinction between the contradicting gravitation models they propose. It is bent space in the first case, information on matter in the second. Gravitation (which present quantum theory is not able not consider on particle level, but dynamic quantum understanding can [21] [23]) between two neutrons and two spheres of the size of the Earth increases by a factor of $10^{114}(!)$. Gravitation in the form of information (on matter) could handle changes over so many orders of magnitude, as well as small fluctuations within (e.g. apparently observed in LIGO experiments), not however a bent space, bent over 114 orders of magnitude (!) around a sphere. Apart from the impossibility to imagine such an immensely fine grading of still measurable space bending around a sphere, a bent space would provide analogue signals, which, as experience shows, can practically only be measured within $0,01 \%$ of the maximum signal.

- Present quantum physics does not allow spreading, propagating photons to produce entropy. They can only lose energy when colliding or encountering a gravitation field. In contrast, dynamic quanta can respect the entropy law for spreading radiation [23] [26], which Einstein still compared with the entropy of an expanding gas to propose the photon concept in analogy to gas particles [28]. This means that the presently claimed cosmological redshift of starlight (attributed to an expanding empty (!) space) may in part simply be entropy generation by expanding, propagating radiation with corresponding consequences for a theory on space dynamics and the origin of the universe [23] [26].

- Due to a dynamic energy, entropy generation is a rate limiting process (relation (8) with (7)). This not only, as shown above, explains the second law of thermodynamics, but also allows derivation of maximum entropy generation (within the given restraints) as entropy law for self-organization processes within non-linear irreversible thermodynamics. Evolution of life would follow such an aim (which is presently denied), which is however subject to and controlled by the superposed genetic code [25]. This explains evolution towards higher complexity and intensifying symbiotic coexistence, as well as the vitality and determination of life as a strive for increasing energy (entropy) turnover (with accompanying increasing local build-up of order and structures) [26]. Such a result, indicating an aim in evolution, is in strict conflict with present understanding of biological mechanisms and origin of intensive controversy (e.g. [29]). Evolution towards maximum entropy generation as an inbuilt aim is also applicable to galactic structures such as, for example, black hole-quasar associations [26].

- Information, which has an energy content, and is contained in biological structures, can, in an irreversible world, also self-organize via feedback processes. This leads to consciousness and spirit [26] with the necessary much more complex information structure. These phenomena could not be explained up to now, which has been criticized, by US philosopher Thomas 
Nagel, as a serious weakness of the present natural scientific world model [30]. Possible implications of a universe, controlled by information (in form of gravitation) and aiming at evolving spirit, for evolution of humanity is discussed in [26].

At the beginning, it was mentioned that quantum theory links energy and entropy with time via the uncertainty relation [6]. What is the ultimate meaning of this relation between energy and time? Because of a so far assumed fundamental time neutrality of quantum states, and since time entered the Schrödinger equation as clock-time via external perturbation theory, the exact meaning of time-energy uncertainty is still debated in spite of significant efforts undertaken to better understand it [31]. The here presented dynamic interpretation of energy and time provides new viewpoints for discussion. One is that the uncertainty relation in quantum physics has to be interpreted differently, because it reflects a dynamic, not a time-neutral phenomenon [26]. The second is that energy and time are indeed linked because free energy generates time via turnover of action, energy times time, and erasure of information on the way to entropy formation (5) (8). Clock-time of quantum physics, however, is not the real dynamic time. It is just a frozen scale.

\subsection{A Way to Better Understand Energy, Nature and the Universe}

In his famous "Lectures on Physics" Richard Feynman emphasized that "...in today's physics we have no knowledge what energy actually is. (...) It is an abstract thing, that does not tell us the mechanisms and reasons for the different formulas (it fulfills)". A look at formula (3) for energy may, superficially seen, support this impression. The here presented approach can, however, show the way to an appropriate answer. Energy behaves similar to money, which, in an economy, also provides for goods and services and involves information turnover (Equation (8)). Money does not need to be handled and used physically in form of materials such as metal or paper. It equally well works in form of pure information (e.g. via credit cards, crypto-currencies). Energy functions basically also as a product of information and information technology in nature. This can readily be deduced from Equation (8). It is the turnover of information $\left(I_{\text {act }}\right)$ and the natural law implementing this, which is facilitating the function of useful, free energy (similar to the function of money and information within a financial economy [26]). Since action time (9) itself is related to information, the saying, from Benjamin Franklin, "time is money", has also a deeper significance. It means that energy turnover is related to time flow, which is actually expressed by relations (4) and (8).

Can the here developed dynamic energy concept now tell the reason for its most important formula, energy conservation? It can! Valuable, free energy works via stored information, countable in bits (8). Exactly this energy for bits $(\mathrm{kTln} 2)$ stored in information appears again as entropic energy (heat) when 
erased. Alternatively, information can, during energy conversion, first be transferred to other (valuable) energy carriers, which store part of the information in between, and be erased later.

A concept of nature with an energy-driven time not only explains the second law of thermodynamics and yields the entropy law for irreversible thermodynamics (subchapter 2.9.), but also recognizes information as a key element for understanding general creativity in nature. Information is involved in feedback processes and the build-up of local inorganic and organic order at the expense of entropy generation and, in form of gravitation, in the dynamics of a quite different universe [23] [26]. In it, time does not move forward or backward homogenously along a given lane or alley as an intricate part of a four-dimensional space-time. Contrary to this, time, as explained here, is the result of an energydriven information erasure, which we register as a flow of action. It generates, what we experience as proceeding time and only develops in the direction we experience within a classically understood space. It is for this reason that invariance against displacement in time in positive and negative direction, as hitherto assumed, cannot and does not work. Time (clock-time), within a space-time constructed by it, is itself an illusion and gives a misleading impression of natural processes and nature whenever energy turnover is involved and wherever irreversibility cannot be neglected for simplification.

With the concept of a fundamentally irreversible nature based on an energydriven time-arrow it is not only easily possible to allocate and accommodate many natural phenomena in a consistent scheme, as the above discussion shows. It would, for the first time, also be possible to say what nature actually is [26]: "Nature is the self-realization of energy through time": Energy self-organizes to matter. Matter self-organizes to inorganic structures and life. Information, provided and active in evolved structures, self-organizes to consciousness and spirit. This appears to be a surprising, but plausible aim for a universe, which is essentially controlled by information.

There is another precise, far reaching new conclusion to be drawn for a fundamentally irreversible nature: With the given explanations for energy (a product of information, like money) and the function of nature (self-realization of energy through time) the universe could not have originated from a chaotic explosion (Big Bang), forced to yield a high entropy, low information product. It must have started from information, which is the essence, the drive of available, free energy, which also generates what we experience as natural reality and, most important, as passing time. This result attributes the origin of the universe to an entirely logic mechanism in itself, which is even understood that way by larger religions (information, as included in words) [26]. The information contained in valuable energy is gradually erased, while creating the large diversity of inorganic and organic structures in the universe and leaving a trace of abandoned, erased information, which we are experiencing as time flow. Without information at the beginning no start and flow of time (action time (9)) would have been 
possible either.

It also becomes understandable why it just was time-neutral fundamental physics that conduced to a chaotic, explosive start of the universe: entropy production and energy loss by spreading photons, like in a spreading gas (as still in comparison discussed by Einstein to justify the particle concept of light [28]), could, as a fundamentally irreversible process, not be properly considered (as redshift) in present time neutral quantum physics.

Numerous now well accepted theoretical concepts will have to change to be compatible with a fundamentally irreversible nature while most experimentally relevant present knowledge will stay as tolerable approximations to time neutrality, proximity to equilibrium and absence of feedback conditions.

To find the right concept of time is a key to understanding nature and the universe. Questioning time neutrality and time symmetry appears to show the way to a more intelligent and ultimately better understandable universe.

\subsection{Conclusions}

The natural philosopher Heraclitus (520-460 BC) did not only observe that "everything changes and nothing stands still", he also said, that "time is a game played beautifully by children". Did he mean that time is at the origin of creativity and imagination? Is time part of and generated out of changes, as discussed above? It seems that such an impression of time as a source of diversity has been communicated again and again in literature sources. In the Indian Sanskrit epic Mahabharata from around $200 \mathrm{BC}$ one can read: "time makes all things grow and destroys all". Such a view survived the time-neutral concepts of scientific progress. The Argentinian writer and philosopher Jorge Luis Borges commented: "Time is the substance I am made of. Time is a river that carries me along, but I am the river". Indeed, energy turnover in the human body is part of time flow, it is the river mentioned by Borges. Swiss writer-philosopher Ludwig Hohl also considered time as a source of unfolding vitality [32]. As a consequence, he expected that exclusion of such a "creative" time from natural science should yield a one-sided development of knowledge. Present science and technology, in spite of ongoing acceleration of research and progress, have indeed been criticized for not fulfilling essential expectations for socially and environmentally relevant progress. As a consequence, a reorientation was called for by experienced philosophers [33]. The late Japanese physicist-psychologist-philosopher Masanao Toda made in this regard a quite precise scientific comment: "Our intuition screams out that time is something that flows, unlike physical time, which is frozen".

The here presented work shows that time is not at all an illusion, just a frozen, neutral scale, along which systems move, but indeed the origin of changes and creativity in nature. It is the dynamics and erasure of information as expression of energy turnover, of feedback reactions and of creativity in nature, which are shaping an ever-changing nature and universe. The here given formalism and 
analysis of the consequences from such a concept has shown that our scientific understanding and world view would open new perspectives while simultaneously becoming more elegant, more rational, simpler and more future oriented. The easy derivation of thermodynamic laws and a more reasonable understanding of evolution promise important new insights. In the field of practical progress, it may be expected that environmentally benign materials and technologies, which evolution has so successfully applied via irreversible processes, may become more accessible and imitable in the long term. The finding that information plays a fundamental role in nature and that self-organization of information yields consciousness and spirit may also stimulate new intellectual ideas about the origin and destination of the universe. But ahead of that a critical confrontation with highly developed, established concepts and theories basing on "timeneutral" physics is inevitable. This paper has been written to provide physical-theoretical arguments for a serious discussion. An entirely irreversible nature around us may underline the need for such a controversy.

\section{Conflicts of Interest}

The author declares no conflicts of interest regarding the publication of this paper.

\section{References}

[1] BBC Presentation and Video (Twitter) by Katie Robertson: 10 February 2020: Are You Experiencing Time Wrong?

[2] Boltzmann, L. (1872) Sitzungsberichte der Akademie der Wissenschaften, 66, 275-370.

[3] Morimoto, T. (1963) Journal of the Physical Society of Japan, 18, 328-331. https://doi.org/10.1143/JPSJ.18.328

[4] Prigogine, I. and Stengers, I. (1997) The End of Certainty: Time, Chaos, and the New Laws of Nature. Simon and Schuster, New York.

[5] Karakostas, V. (1996) Philosophy of Science, 63, 374-400. https://doi.org/10.1086/289917

[6] Beretta, G.P. (2019) Entropy, 21, 679. https://doi.org/10.3390/e21070679

[7] Noether, E. (1918) Invariante Variationsprobleme, Nachrichten der Königlichen Gesellschaft der Wissenschaften zu Göttingen, Mathematisch-Physikalische Klasse, S. 235-257; English Translation.

[8] Wilkens, M. (2015) Noethers Theorem. http://www.quantum.physik.uni-potsdam.de/teaching/ws2015/qm2/Noether.pdf

[9] Hägele, P.C. (2004) Was hat die Entropie mit Information zu tun? https://www.uni-ulm.de/ phaegele/Vorlesung/Grundlagen_II/_information.pdf

[10] Szilard, L. (1929) Zeitschrift für Physik, 53, 840-856. https://doi.org/10.1007/BF01341281

[11] Landauer, R. (1961) IBM Journal of Research and Development, 5, 183-191. https://doi.org/10.1147/rd.53.0183

[12] Reichenbach, H. (1956) The Direction of Time. University of California Press, Oakland.

[13] Withrow, G.J. (1979) What Is Time? Thames and Hudson, London. 
[14] Withrow, G.J. (1980) The Natural Philosophy of Time. Clarendon Press, Oxford.

[15] Davies, P.C.W. (1974) The Physics of Time Asymmetry. Surrey University Press, London.

[16] Zeh, H.D. (1992) The Physical Basis of the Direction of Time. Springer, Berlin. https://doi.org/10.1007/978-3-662-02759-2

[17] Canales, J. (2016) Physics and the Philosopher: Einstein, Bergson and the Debate that Changed our Understanding of Time. Princeton University Press, Princeton. https://doi.org/10.2307/j.ctvc7763q

[18] Rosenblueth, A., Wiener, N. and Bigelow, J. (1943) Philosophy of Science, 10, 18-24. https://doi.org/10.1086/286788

[19] Young, Ch. (2020) Block Universe Theory: Is the Passing of Time an Illusion? https://interestingengineering.com/block-universe-theory-is-the-passing-of-time-an -illusion

[20] Wick, G. (1972) New Scientist, 53, 261-263.

[21] Tributsch, H. (2016) Journal of Modern Physics, 7, 365-374. https://doi.org/10.4236/jmp.2016.74037

[22] Tributsch, H. (2018) Journal of Modern Physics, 9, 1361-1380. https://doi.org/10.4236/jmp.2018.97082

[23] Tributsch, H. (2016) Journal of Modern Physics, 7, 1455-1482. http://www.scirp.org/journal/jmp https://doi.org/10.4236/jmp.2016.712133

[24] Tributsch, H. (2019) Journal of Modern Physics, 10, 1029-1064. https://doi.org/10.4236/jmp.2019.108068

[25] Tributsch, H. (2018) Advances in Anthropology, 8, 140-174. https://file.scirp.org/pdf/AA_2018082716575292.pdf https://doi.org/10.4236/aa.2018.83008

[26] Tributsch, H. (2020) Time Arrow as Trace of Energy, Logical Key to a Spiritual Universe. MyMorawa, Vienna. https://www.amazon.de/Time-Arrow-Trace-Energy-Spiritual/dp/3990936263

[27] Einstein, A. (1916) Annalen der Physik, 49, 769. https://doi.org/10.1002/andp.19163540702

[28] Einstein, A. (1905) Annalen der Physik, 17, 132. https://doi.org/10.1002/andp.19053220607

[29] Gould, S.J. (1992) Scientific American, 267, 118-121. https://doi.org/10.1038/scientificamerican0792-118

[30] Nagel, T. (2012) Mind and Cosmos, Why the Materialist Neo-Darwinian Conception of Nature Is Almost Certainly False. Oxford University Press, Oxford. https://doi.org/10.1093/acprof:oso/9780199919758.001.0001

[31] Busch, P. (2007) The Time-Energy Uncertainty Relation. In: Muga, G., et al., Eds., Time in Quantum Mechanics, Springer, Berlin, 73-105. https://arxiv.org/pdf/quant-ph/0105049.pdf

[32] Hohl, L. (1984) Die Notizen. Suhrkamp, Frankfurt.

[33] Böhme, G., Van Den Daele, W. and Krohn, W. (1976) Social Science Information, 15, 307-330. https://doi.org/10.1177/053901847601500205 